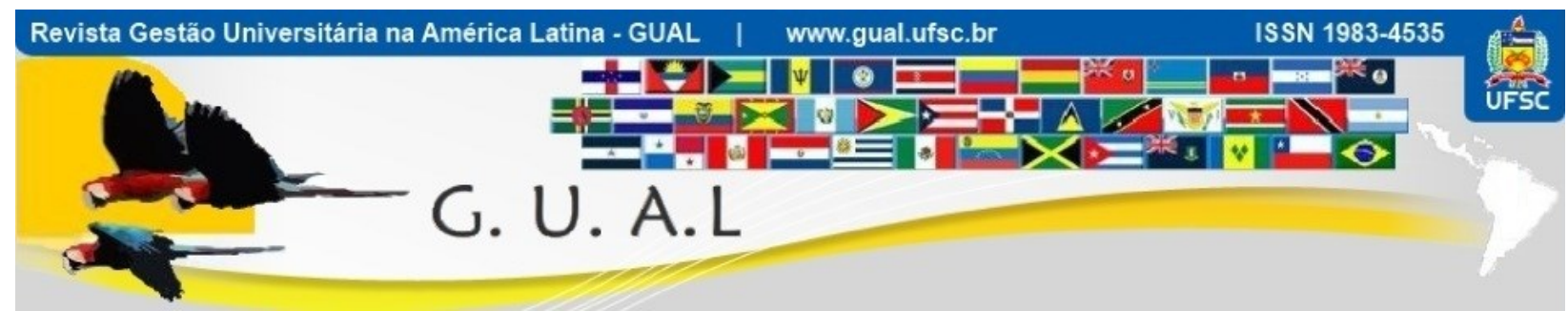

DOI: http://dx.doi.org/10.5007/1983-4535.2020v13n3p256

\title{
PROPOSTA DE FRAMEWORK PARA O PROCESSO DE GESTÃO DE RISCOS NO SETOR PÚBLICO (PROGERIS)
}

\section{PROPOSED FRAMEWORK FOR THE RISK MANAGEMENT PROCESS IN THE PUBLIC SECTOR (PROGERIS)}

\author{
Thiago Oliveira, Graduado \\ https://orcid.org/0000-0001-9744-7551 \\ thiago.oliveira.adm@outlook.com \\ Universidade Federal do Rio Grande do Norte | Programa de Pós Graduação em Administração \\ Natal | Rio Grande do Norte | Brasil
}

Pablo Luan Bezerra Santos, Graduando https://orcid.org/0000-0002-5473-9973 pablobezerra.pj@gmail.com Universidade Federal do Rio Grande do Norte | Curso de Engenharia de Produção

Natal | Rio Grande do Norte | Brasil

Josué Vitor Medeiros Júnior, Doutor http://orcid.org/0000-0002-0361-9744 josuevitor16@gmail.com Universidade Federal do Rio Grande do Norte | Programa de Pós Graduação em Administração Natal | Rio Grande do Norte | Brasil

\section{André Morais Gurgel, Doutor} http://orcid.org/0000-0002-1925-8031 andmgurgel@gmail.com Universidade Federal do Rio Grande do Norte | Programa de Pós Graduação em Administração Natal | Rio Grande do Norte | Brasil

Recebido em 01/abril/2020

Aprovado em 17/julho/2020

Publicado em 01/setembro/2020

Sistema de Avaliação: Double Blind Review
Bruno José Pereira Silva, Mestre https://orcid.org/0000-0003-4157-4898 bruno ufrn_natal@yahoo.com.br Universidade Federal do Rio Grande do Norte $\mid$ Auditoria Interna Natal | Rio Grande do Norte | Brasil

Agradecimento a Universidade Federal do Rio Grande do Norte (UFRN) e a Fundação Norte-riograndense de Pesquisa e Cultura (Funpec) pelo financiamento do projeto de pesquisa Modelo de governança para aquisições em universidades pública, do qual o presente artigo é um de seus resultados. 


\title{
RESUMO
}

Com a intensificação da Gestão de Riscos (GR) no setor público, observa-se a necessidade em se adaptar práticas de modelos reconhecidos em GR para realidade das instituições públicas. O presente trabalho objetiva apresentar a aplicação e os resultados de uma proposta de framework para o Processo de Gestão de Riscos em uma Instituição Federal de Ensino Superior. Esse trabalho é qualitativo e aplicado, sua abordagem metodológica é o estudo de caso e para a coleta dos dados foi adotada a estratégia da triangulação e do grupo focal, observação participante e questionário semiestruturado. Do mesmo modo, essa pesquisa também é descritiva do ponto de vista de seus resultados, sendo os dados analisados na ótica da Análise de Discurso e Análise de Conteúdo. Por fim, os resultados apresentam o detalhamento da aplicação do framework na instituição com o desdobramento de dez etapas sustentadas pela literatura e perspectiva da equipe, demonstrando ainda que o framework trouxe ganhos em relação ao cumprimento dos objetivos organizacionais quanto ao gerenciamento de riscos e aumento da eficiência dos processos.

Palavras-chave: Setor Público. Processo de Gestão de Riscos. Framework.

\begin{abstract}
With the intensification of Risk Management (GR) in the public sector, there is a need to adapt practices of models recognized in GR to the reality of public institutions. This work aims to present the application and results of a proposed framework for the Risk Management Process in a Federal Institution of Higher Education. This work is qualitative and applied, its methodological approach is the case study and for data collection, the triangulation and focus group strategy, participant observation and semi-structured questionnaire were adopted. Likewise, this research is also descriptive from the point of view of its results, with the data being analyzed from the perspective of Discourse Analysis and Content Analysis. Finally, the results present the details of the framework application in the institution, with the development of ten steps supported by the literature and the team's perspective, further demonstrating that the framework has brought gains in relation to the fulfillment of organizational objectives regarding risk management and increased process efficiency.
\end{abstract}

Keywords: Public Sector. Risk Management Process. Framework. 


\section{INTRODUÇÃO}

A Gestão de Riscos (GR) é um tópico recente em pesquisas acadêmicas e principalmente no ambiente corporativo, mostrando-se como um desafio constante para organizações dos setores privado e público (POWER, 2004; PENHA \& PARISI, 2005; KERSTIN et. al., 2014; PRIOTEASA \& CIOCOIU, 2017). Nesse contexto, torna-se nítida a necessidade das organizações na formulação de estratégias sólidas e eficazes, capazes de identificar, avaliar e subsidiar a administração dos riscos (COSO, 2007).

O gerenciamento dos riscos adequado proporciona a melhoria no desempenho dos processos e alcance dos objetivos organizacionais (TSERNG ET AL., 2009; SILVA, 2015). Tais resultados podem ser alcançados por meio de fatores que contribuam com a eficácia na GR, como liderança; apoio da alta administração; educação e capacitações para todos os níveis de organização; suporte de um gerente de risco proativo; comunicação fluída e o uso das informações advindos dos dados do gerenciamento de risco para tomada de decisões (PAPADAKI ET AL., 2014).

Diante disso, diversas abordagens e frameworks recomendados para gestão de riscos já foram desenvolvidos e publicados na literatura para que a GR seja desenvolvida adequadamente em contextos distintos de diversas organizações, de maneira que não há um modelo único possível de ser implementado nesses contextos distintos (POWER, 2004; COSO, 2007; ISO, 2009; IRM, 2018; TUMMALA \& SCHOENHERR, 2011; SILVA, 2015; MORAIS, PINTO \& KLOTZLE, 2018).

Diante de tantas abordagens, percebe-se que não há um modelo detalhadamente desenvolvido que demonstra o como fazer e quais ferramentas utilizar. Naturalmente os modelos apresentam e sugerem a utilização de algumas técnicas, mas não as relacionam com a complexidade do contexto, organização e processos.

No setor público, a GR vem se tornando parte integrante das rotinas de trabalho e dos projetos de melhoria organizacional que visam à qualidade na prestação de serviços (POWER, 2004; LIMA E LEITE, 2011). Consoante essa realidade e impulsionada pela legislação, a Universidade Federal do Rio Grande do Norte (UFRN), com base na Instrução Normativa Conjunta 01/2016 do Ministério do Planejamento, Desenvolvimento e Gestão (MP) e do Ministério da Transparência, Fiscalização e Controle (MTFC), passou a implementar e aplicar o GR no seu contexto. 
Essa iniciativa visa fortalecer a gestão estratégica e governança, por meio da geração de informações e indicadores de risco, assegurando a aderência legal e o auxílio à tomada de decisão que é a base para a governança eficaz (WEINSTEIN E MCWHA, 2008; MP E MTFC, 2016; UFRN, 2017).

Portanto, o presente trabalho objetiva apresentar a aplicação de uma proposta de framework para o processo de gerenciamento de riscos, denominado PROGERIS, em uma IFES. Com isso, é demonstrando o detalhamento do seu passo a passo, paralelamente com a avaliação e validação desse framework junto aos atores dos processos que passaram pela GR para se responder a seguinte questão: como o framework de gerenciamento de riscos proposto pode apoiar o ciclo de gestão de riscos na IFES?

Assim, a seguir serão abordados: os conceitos de GR e seus principais modelos consolidados, bem como benefícios e limitações; o desenvolvimento do gerenciamento de riscos em IFES; a metodologia utilizada para o desenvolvimento do framework; o detalhamento das etapas e avaliação do framework aplicado na IFES; as considerações quanto à validação dessa metodologia; limitações da pesquisa e perspectivas de trabalhos futuros.

\section{GESTÃO DE RISCOS}

Após a crise financeira ocorrida em 2008, as organizações mudaram a forma de como gerenciavam seus riscos, saindo de uma perspectiva tradicional que dá ênfase nos riscos advindos da área financeira (MORAIS; PINTO \& KLOTZLE, 2018) para uma abordagem mais geral, ampliando seu escopo e trabalhando com riscos que estão em toda a estrutura corporativa (GUN et. al., 2013; KERSTIN et. al., 2014), pois é notório que em toda a estrutura, sistema, processo e atividade de uma organização envolve riscos (ISO, 2009; IRM, 2018).

O movimento da gestão de riscos caminha em paralelo com Governança Corporativa, principalmente no setor público (MIRANDA, 2017; SALES, 2018). A preocupação central na GR é o dever de assegurar o alcance dos objetivos organizacionais (ÁVILA, 2014). Nessa perspectiva, os controles internos assumem um papel fundamental no contexto desse gerenciamento, visto que esses avaliam o alcance da missão organizacional (BEUREN; ZONATTO, 2014).

Assim, a Gestão de Riscos é caracterizada como um elemento-chave da governança nas organizações e precisa se encaixar adequadamente como um processo de gestão. Esse 
processo deve ser associado a todo o processo decisório e ao estabelecimento e alcance dos objetivos estratégicos (MIRANDA, 2017).

Diante disso, ações e atividades de controle são refletidas no contexto organizacional. Essas iniciativas são elementos de gestão preventivos e detectivos referentes a planos de ação e contingência, em resposta a possível materialização de ameaças (ENAP, 2018).

A gestão de riscos pode ser definida como um processo contínuo capaz de identificar e mitigar elementos e eventos responsáveis por maximizar efeitos negativos nas práticas organizacionais, interferindo no alcance de seus objetivos (PENHA \& PARISI, 2005; COSO, 2007; SANTOS \& COELHO, 2018). Esses eventos podem gerar impactos negativos, positivos ou ambos simultaneamente.

Os eventos que geram impactos negativos representam riscos que podem impedir o alcance de objetivos e criação de valor (COSO, 2007). Além disso, há também eventos que geram impactos positivos. Esses são avaliados e mensurados em termos de probabilidade de acontecimentos e impacto. (SARKA \& DUTTA, 2011)

Dessa forma, o gerenciamento de riscos deve ser aplicado em todas as organizações, em suas diversas áreas, níveis e contextos, a qualquer momento, bem como funções, projetos, atividades e práticas específicas (IRM, 2018). No setor corporativo em geral, a GR tornou-se percebida como parte integrante dos negócios e estratégias organizacionais (POWER, 2004).

As organizações habitualmente gerenciam os seus riscos identificando-os, analisandoos e tratando-os para posteriormente mantê-los compatíveis com o apetite a risco da organização e garantindo razoavelmente o cumprimento dos seus objetivos, satisfazendo seus critérios de aceitabilidade (COSO, 2007; ISO, 2009). Os riscos que podem gerar impactos positivos são tradados como oportunidades e são analisados em mensurados para que seus benefícios sejam potencializadores da missão e alcance dos objetivos dessas organizações.

Além disso, há constantes debates sobre os benefícios estratégicos para organizações resultantes de uma gestão de risco tratada de maneira mais explícita, ou seja, envolvendo toda a organização (TSERNG ET AL., 2009; SILVA, 2015). Diante disso, há uma necessidade em se ter modelos estruturados, levando em consideração a complexidade das organizações, para que a GR possa ser bem desenvolvida e traga ganhos e valor agregado.

O Enterprise Risk Management (ERM), é uma estrutura que fornece definições, conceitos e princípios que apoiam a construção e desenvolvimento do gerenciamento de riscos coorporativos $(\mathrm{COSO}, 2007)$. Essa estrutura também dá subsídio às organizações na 
verificação do seu gerenciamento de riscos, demostrando se esse é eficaz e quais parâmetros e diretrizes precisam ser estabelecidos para que essa estrutura assim seja (COSO, 2007; SILVA, 2015).

Deste modo, os componentes de gerenciamento de riscos definidos por (COSO, 2007) são: Ambiente; Fixação de Objetivos; Identificação de Eventos; Avaliação de Riscos; Resposta a Risco; Atividades de Controle; Informações e Comunicações; e Monitoramento. A Figura 1 a seguir apresenta a estrutura dos 8 componentes interrelacionados, pelos quais a gestão organizacional está integrada com o processo de gestão de riscos (COSO, 2007):

Já a ISO 31000 (2009), de maneira geral, fornece políticas e diretrizes bem definidas sobre como se planejar, implementar, gerenciar recursos para o gerenciamento dos riscos e monitorar, disponibilizando informações da GR para a alta gestão e partes interessadas. Esse alinhamento fornece o suporte necessário à criação de um sistema de gerenciamento e controle eficaz de riscos (IRM, 2018).

Ainda assim, a normativa estabelece um determinado número de requisitos que precisam ser satisfeitos para uma GR adequada (ISO, 2009; IRM, 2018), sendo eles: Estabelecimento do contexto; Comunicação e consulta; Processo de avaliação de riscos; Identificação dos riscos, Análise e Avaliação de riscos; Tratamento de riscos, Monitoramento e Análise crítica.

Percebe-se que o COSO (2007) e a ISO (2009) estabelecem políticas, diretrizes e boas práticas no gerenciamento dos riscos. No entanto, não apresentam um passo a passo ou checklist explícito que deva ser seguido à risca em suas implementações, pois o objetivo desses dois modelos não é a prescrição de um sistema de gerenciamento de riscos e sim em auxiliar a compreensão e implementação da GR nos diversos contextos organizacionais (SILVA, 2015).

Não há, também, um detalhamento de como desenvolver cada uma das etapas destes dois modelos; o como fazer, e quais ferramentas utilizar para tanto. Nota-se também que o tratamento dos riscos propriamente dito, ou seja, a execução dos planos de ação e mitigação não é adequadamente abordada e aprofundada, levando em consideração a sua importância no gerenciamento dos riscos.

Desse modo, percebe-se que a maneira como essas ações serão postas em execução fica a critério da organização. Nessa perspectiva, nota-se que as organizações devem estudar e 
adaptar esses modelos de gerenciamento de riscos ao seu contexto específico e de acordo com as suas necessidades (SILVA, 2015). Então, o desafio para os gestores e profissionais que pretendem trabalhar com a GR é reorganizar essas práticas, construindo uma abordagem que mais se adeque a sua realidade.

É interessante destacar que o modelo deve apresentar capacidade de aplicabilidade em todos os níveis da organização, estabelecendo um sistema de gerenciamento de riscos eficaz (ISO, 2009). Assim, esse modelo de gerenciamento de riscos deve propiciar uma visão holística de todos os riscos da organização (PENHA \& PARISI, 2005; KAPLAN \& MIKES, 2012).

\section{GESTÃO DE RISCOS NAS INSTITUIÇÕES FEDERAIS DE ENSINO SUPERIOR (IFES)}

As Instituições Federais de Ensino Superior (IFES) tem uma estrutura complexa e suas funções básicas, ou processos finalísticos dentro da sua cadeia de valor, consistem na oferta de ensino, pesquisa e extensão bem como a oferta de produtos e serviços com foco na inovação, que possam contribuir para a qualidade de vida e desenvolvimento da sociedade, através do conhecimento gerado (BOLZAN, 2006)

Ainda segundo Bolzan (2006), no ambiente interno das IFES é contínua a busca da gestão pela qualidade dos processos, tanto acadêmicos como também administrativos, para se alcançar uma prestação de serviços e gestão universitária adequada e eficiente, com iniciativas que resolvam as suas falhas.

Recentemente, a GR tornou-se uma preocupação crescente para os gestores das IFES que estão sendo impactados por uma pressão crescente em torno da qualidade na gestão, transparência e responsabilidade dos governos federais, estaduais e órgãos de controle. Pesquisas recentes demonstram que, além das universidades já estarem adotando práticas de GR, há uma construção e padronização de guias e boas práticas para orientação na implementação da GR nessas instituições (WEINSTEIN \& MCWHA, 2008; SILVA, 2015).

Em 2018, o Tribunal de Contas da União (TCU), realizou um levantamento denominado Auditoria Exposição da Administração Pública Federal a Fraude e Corrupção, com o objetivo de averiguar se os mecanismos de prevenção e detecção relacionados a fraude e corrupção de IFES estão compatíveis com capital e de regulação. Dentre os indicadores calculados, destaca-se o índice de fragilidade de controles na gestão de riscos e controles internos, cujo valores variam de 0 a 1 , sendo os menores valores os que indicam a menor 
fragilidade nesses controles. Avaliou-se 105 IFES, das quais apenas 15,24\% possuem índice entre 0.3 e 0.6 , enquanto $70,48 \%$ possuem índice entre 0.6 e 0,85 e $14,28 \%$ entre 0.85 e 1 (TCU, 2018).

Vale pontuar que nas universidades e no setor público em geral, a GR vem se tornando parte das rotinas de trabalho, com sua abordagem orientando as instituições a reverem o modo como desempenham suas atribuições e buscam a melhoria contínua na ausência de mecanismos de mercado que a impulsionem a ter excelência (POWER, 2004; WEINSTEIN \& MCWHA, 2008).

Logo, percebe-se que as IFES devem estar alinhadas com os benefícios dessa abordagem e precisam manter e revisar controles internos de gestão, tendo como base a identificação, avaliação e o gerenciamento dos riscos, desenvolvendo controles adequados para mitigar a probabilidade de ocorrência e impactos dos riscos nos seus processos (MP E MTFC, 2016). Diante disso, a GR pode ser considerada a base para iniciativas que resultem na melhoria e qualidade dos serviços públicos (POWER, 2004, SILVA, 2015).

Destarte, a adesão de práticas de GR, aliada aos controles internos nessas instituições é requisito fundamental para o eficiente funcionamento das organizações públicas. Não obstante, por se tratar também de atividade que apoia o alcance dos objetivos e agregação de valor, fica evidente o papel da auditoria interna nesse gerenciamento, atuando na verificação desses controles, no sistema de governança e gestão dos riscos (SALES, 2018).

Com isso, percebe-se que um modelo de gestão de riscos precisa ser desenvolvido a luz do contexto da organização e deve ter como apoio ferramentas bem definidas que facilitem e suportem todo o processo do gerenciamento dos riscos neste contexto.

\section{METODOLOGIA}

Este trabalho é de natureza qualitativa (GIBBS, 2009; SAMPIERE, 2013). Sua abordagem metodológica é estudo de caso, já que há um interesse em se entender fenômenos de casos complexos (YIN, 2005). Assim, este trabalho baseia-se no caso da implantação da GR em uma IFES.

Essa pesquisa também é aplicada, a qual possui um caráter participativo na produção e uso de conhecimentos, permitindo um processo de mudança bem estruturado para a promoção de ações transformadoras (THIOLLENT, 2011). Do ponto de vista dos resultados, a presente pesquisa também é descritiva (RICHARDSON, 2008). 
Quanto aos procedimentos metodológicos, inicialmente, como orienta a ISO 31000 (2009) e Silva (2015) foram desenvolvidas pesquisas documentais para se conhecer os modelos de GR que são utilizados nas corporações e adequá-los no desenvolvimento de um framework próprio para a instituição em evidência.

Conquanto, foram estabelecidas etapas para o processo de gerenciamento de riscos com base nos modelos apresentados, sendo a maioria das etapas baseadas na ISO 31000 (2009) e, em cada etapa, foram adotadas ferramentas da administração e engenharia de produção, adaptadas a este contexto.

Após isso, o framework foi formalizado apresentado à alta gestão da IFES e posto em uso. Paralelamente a sua implantação e para que se obter informações detalhadas quanto ao passo a passo do framework e diminuir as contradições, foram realizadas atividades de Observação Participante (OP) e Grupos Focais com registro de informações quanto a operacionalização desse processo para a ocorrência de melhorias contínuas no modelo, bem como a aplicação de um questionário online para que alguns colaboradores da IFES pudessem avaliar se, e como, o PROGERIS trouxe eficiência na gestão dos riscos da instituição.

No total foram 22 respondentes desses questionários em 9 diferentes setores da IFES, 10 entrevistados e diversos participantes dos grupos focais. Esses encontros foram gravados e posteriormente transcritos totalizando 2 páginas de transcrição. Nos resultados, as falas de alguns entrevistados são apresentadas.

Após a transcrição, inicialmente foi utilizada a Análise de Discurso (AD) para se ter uma primeira interpretação, bastando que sua materialidade produzisse sentido para a interpretação, sendo uma análise vertical (CAREGNATO \& MUTTI, 2006). Assim, diante dessa primeira análise foram elencados alguns trechos mais relevantes para essa pesquisa que, posteriormente, passariam por um tratamento mais profundo.

Num segundo momento foi utilizada a Análise de Conteúdo (AC), compreendendo a exploração do material já separado na primeira análise, categorização de alguns trechos mais relevantes e o tratamento e interpretação dos desses trechos, ocorrendo a associação de inferências para a obtenção dos resultados mais consistentes da pesquisa (BARDIN, 2006; RICHARDSON, 2008; VERGARA, 2015).

Além disso, a medida em que o framework fora implementado na IFES, planilhas de controle foram sendo alimentadas para se ter um controle contínuo sobre a GR desenvolvida. Nessas planilhas foram registrados: setores, processos de trabalho dos setores e priorização, 
mapas de riscos com eventos de riscos identificados nos processos e seus respectivos níveis de riscos, planos de ação que foram desenvolvidos para a mitigação dos riscos, responsáveis por executar esses planos de ação e riscos residuais, ou seja, o nível de risco que ainda restou após a implementações das ações (SILVA, 2015).

Assim, os dados dessas planilhas de controle também foram interpretados com técnicas de estatística descritiva simples e são apresentados nos resultados. Além disso, foi construído e aplicado, para os 22 participantes de diferentes setores, um Net Promoter Score (NPS) online para se avaliar do nível de satisfação (HAMILTON ET AL., 2014) e o quanto os colaboradores que utilizaram o framework recomendam o mesmo aos demais da IFES. Para essa medição foi elaborada o seguinte questionamento: Em uma escala de 0 a 10, o quanto você recomendaria o PROGERIS para os demais setores da instituição?

Por fim, após todas essas análises, os resultados foram obtidos quanto à aplicação do PROGERIS na IFES e é apresentado a seguir, sendo essa implantação respaldada tanto pelos discursos como pelos dados estatísticos quanto a esse gerenciamento.

\section{FRAMEWORK PARA O PROCESSO DE GESTÃO DE RISCOS (PROGERIS)}

\subsection{APRESENTAÇÃO E DETALHAMENTO DO PROGERIS}

Inicialmente foi concebida a estrutura de GR da instituição com: a constituição do Comitê de Riscos, Governança e Controles, que reuniu a alta gestão da Universidade para a constante validação e alinhamento com expectativas administrativas e legais; a aprovação do plano (metodologia) de gestão de riscos; a definição de qual setor iria desenvolver a implementação da GR; as formas de comunicação para o alinhamento estratégico e governança do gerenciamento dos riscos; e o sistema de informação que apoiaria o monitoramento e controle dos riscos (WEINSTEIN \& MCWHA, 2008).

Após essa definição, uma proposta de framework foi desenvolvida para que a instituição desenvolva sua GR de forma adequada e eficiente através de um processo bem definido. Esse framework é denominado PROGERIS e seu processo, constituído por 10 etapas, é apresentado na Figura 4 a seguir: 
Figura 1 Estrutura de Gestão de Riscos UFRN

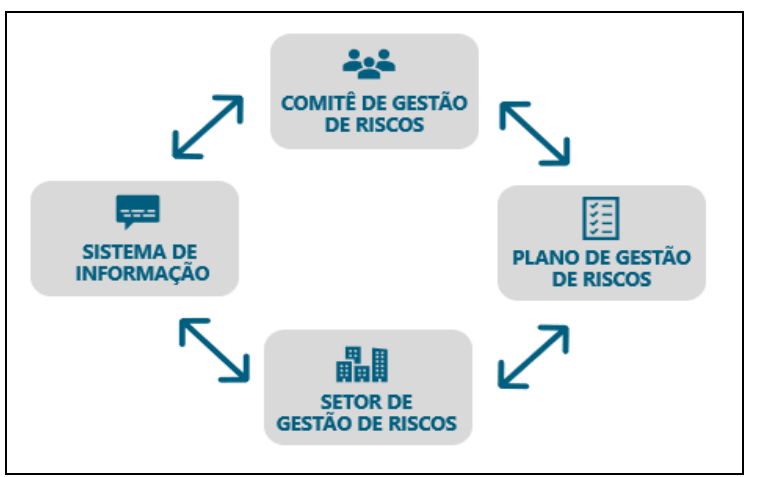

Fonte: Elaboração própria (2019).

Figura 2 Framework de Gestão de Riscos

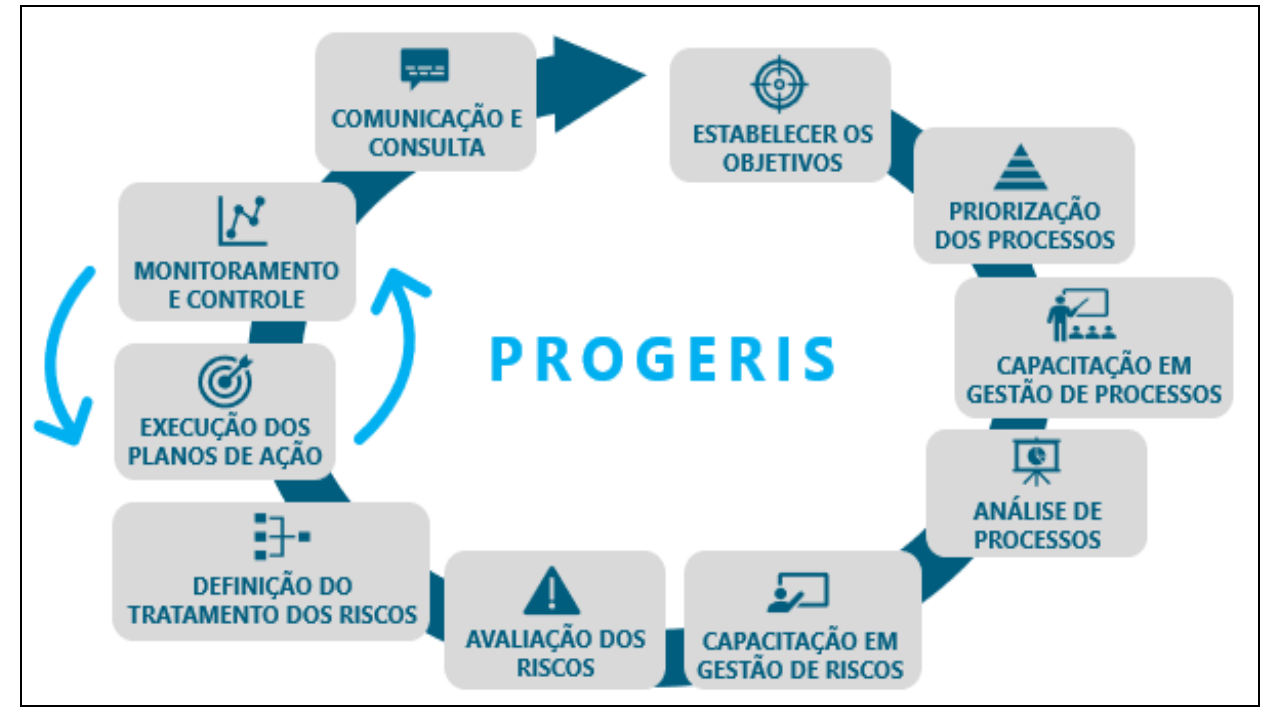

Fonte 1: Elaboração própria (2019).

A Gestão dos riscos a ser desenvolvida na IFES tem como objetivo mitigar os riscos que podem impedir ou dificultar o alcance dos seus objetivos (UFRN, 2017). Nessa ótica, a Cadeia de Valor da IFES foi elaborada, para a identificação e priorização dos Macroprocessos finalísticos e os de apoio que seriam analisados à luz da gestão de riscos (ABPMP, 2013).

Após essa elaboração, foi desenvolvida a primeira etapa do framework que se refere a Estabelecer os Objetivos da gestão dos riscos da IFES. Ressalta-se que essa etapa foi extraída do COSO (2007) e da ISO (2009). Logo, foi feita uma priorização diante os 67 macroprocessos da IFES e definiu-se que a GR abrangerá toda a instituição em cinco anos (UFRN, 2017), conforme exposto na Tabela 1, sendo os objetivos da GR alinhados aos objetivos estratégicos da IFES (KAPLAN \& MIKES, 2012). 
Tabela 1 Objetivos da Gestão de Riscos na UFRN

\begin{tabular}{c|c}
\hline \hline Ano & Quantidade de Processos com Riscos Gerenciados \\
\hline 2018 & 14 \\
\hline 2019 & 14 \\
\hline 2020 & 13 \\
\hline 2021 & 13 \\
\hline 2022 & 13 \\
\hline \hline
\end{tabular}

Fonte: UFRN (2019).

A segunda etapa do framework é a Priorização dos Processos. Com os macroprocessos já identificados na cadeia de valor, os analistas de riscos verificam quais são as Unidades Institucionais responsáveis pelos Macroprocessos que serão abordados de acordo com os objetivos.

Tabela 2 Trechos das entrevistas

Entrevistado 1: (...) é importante priorizar porque muitas vezes um setor é muito grande e não tem como 'abraçar' todos os processos...

Fonte: Dados da pesquisa (2019).

Após isso e levando em consideração que os macroprocessos podem ser constituídos por diversos processos e sub processos (ABPMP, 2013) é verificado quais são os processos principais, ou finalístico, e os de apoio que essa unidade executa. Dessa forma, se pode identificar quais são os processos que devem ser analisados na ótica de GR.

A partir dessa análise e priorização desses processos, o cronograma de ação para a equipe de execução da GR é elaborado e a terceira etapa do processo pode ser desenvolvida. Essa se refere à Capacitação em Gestão de Processos. Os atores dos processos, a fim de facilitar a compreensão e execução da próxima fase e capacitar esses recursos humanos, são habilitados/instruídos sobre os conceitos básicos de processos, estrutura funcional da IFES, notação para processos de negócios e importância dela na rotina de trabalho.

Feito isso, dá-se prosseguimento à fase de Análise dos Processos, que é composta por 2 sub etapas: mapeamento dos processos; identificação dos problemas e propostas de melhoria. De forma simples, os atores dos processos participam de oficinas para ativamente mapearem estes, detalhando o seu passo a passo, inputs e outputs, recursos e, por fim, o validarem de forma conjunta e consensual. 
$\mathrm{Na}$ segunda fase, os atores dos processos participam de uma oficina para a Identificação dos Problemas que podem interferir no alcance dos objetivos dos processos, e na Identificação de possíveis Propostas de Melhoria para promover uma maior eficiência do processo e resolução dos problemas também identificados.

Nessa oficina, o processo é apresentado de forma visual para que os seus atores identifiquem, em seu fluxo, os problemas. Ressalta-se que o apoio de ferramentas visuais à gestão aumenta a eficiência na identificação de problemas nos processos (LIKER, 2005). Do mesmo modo, os atores identificam possíveis propostas de melhoria que sirvam para resolver os problemas identificados nesse processo.

A importância da representação dos processos em fluxogramas possui relevância, de acordo com a fala a seguir:

Tabela 3 Trechos das entrevistas

Entrevistado 2: (...) é bastante interessante para gente entender como faz o processo e para o público externo saber o que a gente faz aqui...

Fonte: Dados da pesquisa (2019).

Assim, os problemas são identificados por meio da análise das entradas, tarefas, recursos, responsabilidades e saídas. Também são considerados os fatores internos e externos que podem influenciar no alcance dos objetivos do processo (COSO, 2007).

De forma similar à capacitação em gestão de processos, os atores também são submetidos à Capacitação em Gestão de Riscos. Essa etapa do framework considera que a GR deve ser compartilhada e ser de responsabilidade de toda a organização, todos os níveis e todos os colaboradores, gerando multiplicadores e pessoal capacitado interdisciplinarmente para um bom gerenciamento dos riscos (MIRANDA, 2017). Na capacitação, são explanados princípios básicos e importância da GR na IFES, classificação dos eventos de riscos e mecanismos de controle.

Levantadas as problemáticas e, adicionalmente, com os atores capacitados, é possível então dar início à quarta etapa, que consiste na Avaliação dos Riscos. Nessa etapa a análise de causa e efeito é desenvolvida para identificar se o que foi apresentado como um problema possa ser, na verdade, a causa de algum outro problema mais abrangente. Assim, são evidenciados os eventos que devem ter sua ocorrência controlada para não desencadearem em riscos no processo (KAPLAN \& MIKES, 2012). 
Essa etapa foi extraída do COSO (2007) e da ISO (2009). A identificação dos riscos é parte essencial para um bom gerenciamento; falhas nessa etapa podem causar inadequações em todo o processo afetando criticamente os objetivos organizacionais (RAZ \& MICHAEL, 2001; ROSTAMI, 2016).

Após a conversão dos problemas em eventos de riscos, é feita uma breve validação com os atores dos processos e seus gestores. A próxima etapa é iniciada com a mensuração da probabilidade e impacto desses eventos de riscos, novamente com todos os atores. Para isso, à probabilidade e ao impacto são determinados pesos de 1 a 5 , onde 1 representa um grau muito baixo e 5 muito alto. Os executores e gestores dos processos utilizam uma tecnologia de votação para classificar cada risco, na escala apresentada (KAPLAN \& MIKES, 2012).

Feito isso, multiplica-se essas duas variáveis, probabilidade e impacto, e se tem o produto dos eventos de riscos, classificando-os em Insignificante, Pequeno, Médio, Grande e Crítico (UFRN, 2017). Para essa classificação, utiliza-se como referência a matriz de riscos do Plano de Gerenciamento de Riscos da Instituição (UFRN, 2017), a qual demonstra o nível de apetite ao risco da organização (MIRANDA, 2017), apresentada na Figura 5 a seguir:

Figura 3 Matriz de Riscos da UFRN

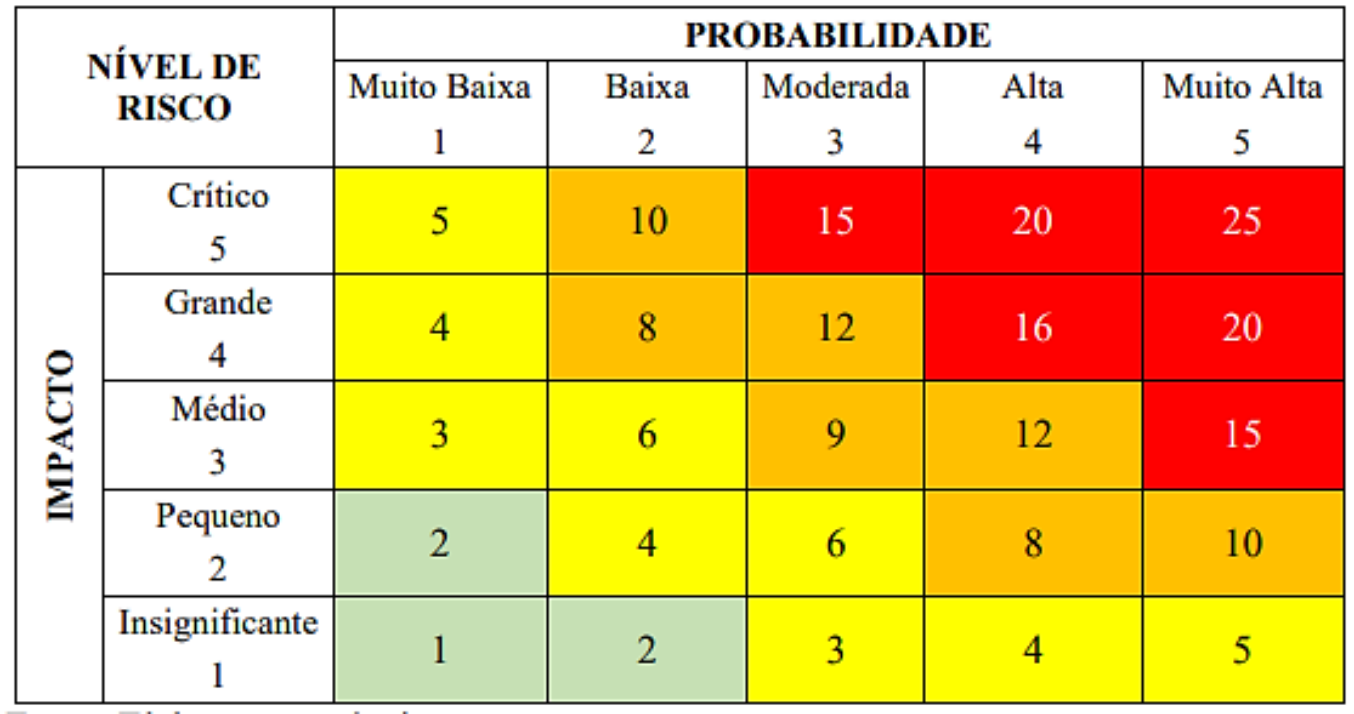

Fonte 2: UFRN (2017).

Ainda nesta etapa, destaca-se a importância dos facilitadores externos à unidade para melhor levantamento e classificação dos eventos de riscos. 
Tabela 4 Trechos das entrevistas

Entrevistado 2: (...) facilitou o diálogo da gente [a colaboração dos facilitadores] ...

Entrevistado 1: (...) se a gente fosse fazer sozinho, desconhecendo a metodologia a gente poderia errar e isso iria prejudicar todo o levantamento de riscos daquele processo...

Fonte: Dados da pesquisa (2019).

Após todas essas etapas de identificação e classificação dos riscos, os planos de ação podem ser elaborados de forma adequada a realidade dos mesmos (WEINSTEIN \& MCWHA, 2008). Assim, a próxima etapa do processo de GR é a Definição do Tratamento dos Riscos. Essa etapa também foi extraída do COSO (2007) e da ISO (2009).

Pontua-se que as propostas de melhoria que foram levantadas nas oficinas são analisadas, com o objetivo de avaliar se essas propostas podem ser desdobradas em mecanismos de controle dos seus respectivos riscos. A partir disso, são elaborados os respectivos planos de ação para a mitigação e contingenciamento dos riscos junto aos atores dos processos, bem como são definidos os responsáveis por executá-los. É importante garantir que os atores tenham autonomia e liberdade para propor mecanismos de controle.

Tabela 5 Trechos das entrevistas

Entrevistado 1: (...) nós [atores do processo] sabíamos quais eram os problemas e nós tínhamos as respostas ...

Entrevistado 3: (...) foi muito importante porque todos colaboraram. E principalmente a liberdade que vocês [facilitadores] nos deram, porque a gente praticamente propôs tudo, vocês juntaram as informações que nós demos e organizaram aquilo ali...

Fonte: Dados da pesquisa (2019).

Logo após a elaboração e validação desses dos planos de ação junto aos atores dos processos, a etapa 6 consiste na Execução dos Planos de Ação, etapa essa extraída do COSO (2007) e da ISO (2009), a qual são planejados os cronogramas, indicadores e metas para a execução desses planos, sendo priorizados de acordo com a suas classificações.

Para isso, algumas abordagens da Gestão de projetos são utilizadas com foco na simplificação e eficiência na execução desses planos de ação. Assim, nessa etapa a metodologia de Gestão de Projetos é integrada à Gestão de Riscos, para se planejar, executar, controlar e monitorar e encerrar os planos de ação, a fim de mitigar todo e qualquer risco. $\mathrm{O}$ processo de gestão de riscos nunca entregará os prometidos benefícios se a execução dos planos de ação não for desenvolvida de forma eficiente e eficaz (HILLSON, 1999). Ainda 
nessa etapa, observou-se à necessidade de os facilitadores direcionarem de forma mais completa e estruturada a execução dos planos de ação.

Tabela 6 Trechos das entrevistas

Entrevistado 4: (...) muitas vezes a pessoa não está acostumada a usar as ferramentas para resolver aquele problema [risco] ...

Fonte: Dados da pesquisa (2019).

Outro fator importante, destacado pelo Entrevistado 2, relaciona-se às dificuldades para resolver os problemas que não dependem da unidade onde foram aplicadas as oficinas, apesar do engajamento e autogerenciamento da equipe na execução das atividades.

Tabela 7 Trechos das entrevistas

Entrevistado 2: (...) o problema que eu vi na execução são os problemas que não dependem da gente ...

Fonte: Dados da pesquisa (2019).

A próxima etapa framework é o Monitoramento e Controle, que também foi extraída do modelo COSO (2007) e da ISO (2009). Com a devida execução dos planos de ação, os riscos devem ser reavaliados para ser identificado o nível dos riscos residuais. O Risco residual refere-se aos riscos que permanecem após a realização dos planos de ação (ROBERTS, WALLACE \& MCCLURE, 2012). Essa avaliação consiste em verificar se a probabilidade e impacto do risco diminuíram e o plano de ação cumpriu o seu papel (SILVA, 2015).

As organizações também precisam de uma estrutura de monitoramento e controle de risco (KAPLAN \& MIKES, 2012). Vale salientar a importância de ferramentas que facilitem o monitoramento e controle. Além disso, as boas práticas compartilhadas durante a GR serão incorporadas na rotina de trabalho. Esses benefícios são interpretados dos trechos a seguir:

Tabela 8 Trechos das entrevistas

Entrevistado 3: (...) simplificou [o uso das ferramentas] o trabalho para quem não está acostumado com os conceitos de projetos ... (...) começou a ser incorporado para as demais atividades da unidade [ferramenta] ...

Entrevistado 3: (...) pois um consegue ver o andamento do problema do outro.

Fonte: Dados da pesquisa (2019). 
Para o esse monitoramento, o GERIFES.net, sistema de informação, apoia todo o processo e estrutura de GR da instituição, fornecendo uma visão geral do status dos riscos de forma visual, emitindo relatórios gerenciais e dando transparência desse processo à alta gestão universitária.

Ademais, a última etapa do PROGERIS é a Comunicação e Consulta. Com o suporte informacional que o GERIFES.net proporciona, relatórios são gerados quanto ao status da GR são pautados e apresentados em reuniões com o Comitê de Gestão de Riscos. Visto isso, pontua-se que essa etapa também foi extraída do COSO (2007) e da ISO (2009).

As reuniões têm o objetivo de comunicar e consultar a alta administração, para o alinhamento de expectativas e atualização do andamento desse processo (COSO, 2007; ISO, 2009; SILVA, 2015; MIRANDA, 2017).Verifica-se que essas reuniões são pertinentes inclusive para a exposição de alguns problemas que são oriundos de um risco e seu plano e ação pode ter uma abrangência e escopo significativamente maior ao ponto dessa ação se tornar um projeto.

\section{RESULTADOS DA IMPLEMENTAÇÃO DO PROGERIS NA IFES}

A partir da implantação da GR na instituição, que foi iniciada em novembro de 2018, o PROGERIS já foi aplicado em treze macroprocessos da cadeia de valor da IFES, envolvendo um total de 135 subprocessos. Esse resultado gera um input para que os objetivos da GR sejam revistos, pois no decorrer desse gerenciamento foram identificados um número a mais de macroprocessos do que foram fixados inicialmente.

Após análises da planilha onde constam as matrizes de riscos geradas, nota-se que $41,57 \%$ dos eventos de riscos foram classificados como Muito Altos, 25,84\% como altos, $21,35 \%$ moderados e $11,24 \%$ Baixos. Dessa forma, $67,41 \%$ dos riscos classificados estão além do apetite de Riscos determinado na política de Gestão de Riscos da IFES.

Objetivando entender as percepções dos participantes quanto ao uso do PROGERIS na gestão dos seus riscos, aplicou-se um questionário on-line com 22 servidores públicos de 9 setores diferentes da IFES. Os resultados apontam um nível de satisfação e recomendação da metodologia de aproximadamente $95,45 \%$, com o NPS, bem como um nível de entendimento da metodologia acima de 90\%, sendo possível sugerir que a aplicação das etapas do framework possui alta aceitabilidade dos envolvidos. 
Gráfico $1 \%$ de Eventos de Riscos por grau de risco

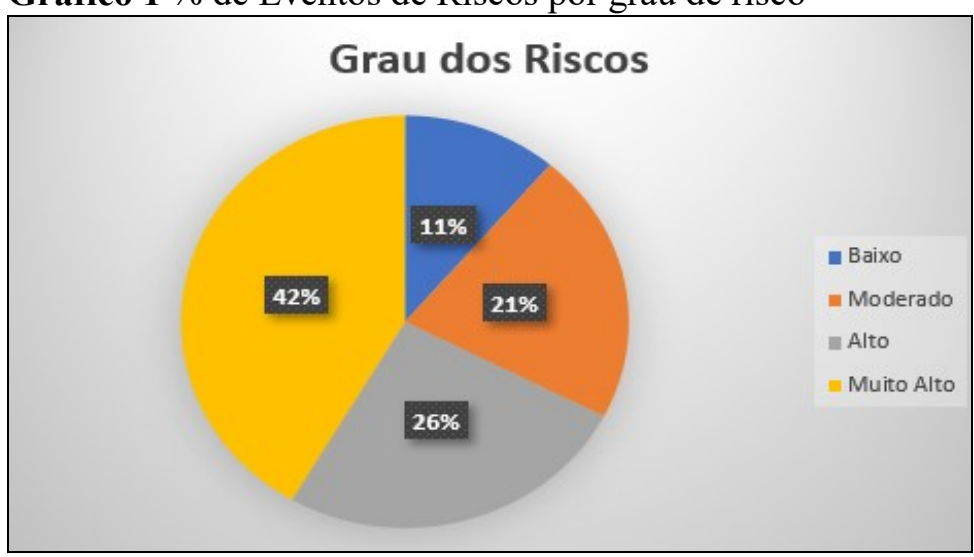

Fonte: Dados da pesquisa (2019).

Gráfico 2 Respostas ao questionário online

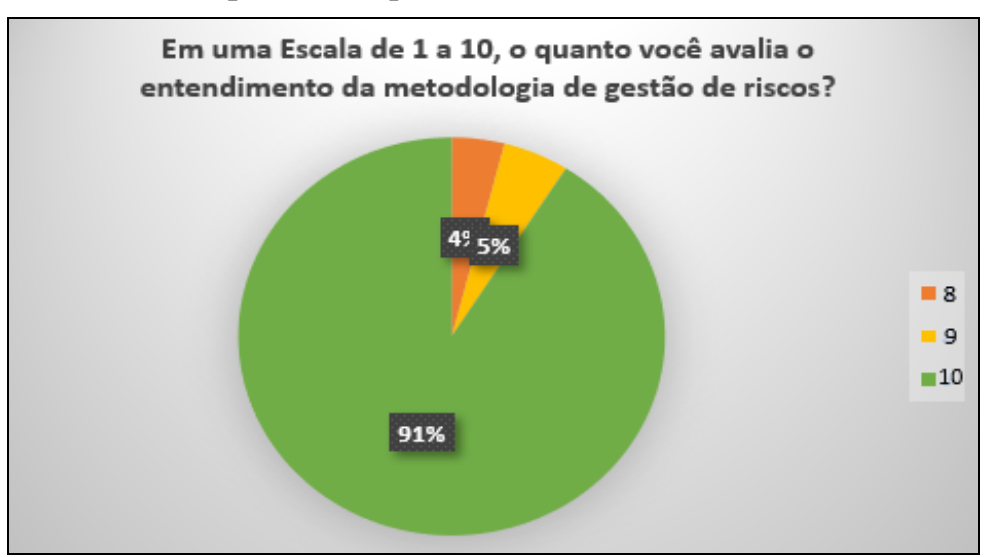

Fonte: Dados da pesquisa (2019).

Diante disso, os resultados parecem indicar que o PROGERIS proporciona maior clareza na rotina de trabalho, além de sistematizar a identificação e resolução de oportunidades de melhoria das unidades

Tabela 9 Trechos das entrevistas

Entrevistado 4: (...) fez refletir mais sobre o processo que eu sou mais responsável [...] foi muito importante pensar e refletir sobre os problemas que a gente passa e que são corriqueiros e que às vezes o setor não tem tempo de pensar e refletir soluções...

Entrevistado 5: (...) foi muito importante para o setor e ajudou a clarear bastante para gente até algumas etapas de processo que a gente passava batido em algumas coisas e identificamos alguns problemas...

Fonte: Dados da pesquisa (2019).

Nesse contexto, o framework proposto apresenta uma série de vantagens em relação às demais abordagens. A princípio, ressalta-se que o PROGERIS pode possibilitar melhoria dos 
processos e mostra-se, também, uma ferramenta para identificação de pontos de melhoria na unidade/setor, com as devidas capacitações e gestão de processos e riscos. Além disso, é importante destacar os ganhos em relação à eficiência e eficácia operacional e engajamento da equipe identificado, culminando na otimização de recursos da instituição e no alcance dos objetivos organizacionais.

Tabela 10 Trechos das entrevistas

Entrevistado 2: (...) o projeto (de gestão de riscos) veio trazer também essa forma da gente entender melhor, de forma mais clara, mais profissional [os riscos]; então, de certa forma, foi bom para gente visualizar isso e tentar desenvolver de uma forma conjunto...

Fonte: Dados da pesquisa (2019).

Por outro lado, também se faz necessário comprovar e/ou validar os benefícios por um olhar ou ponto de vista externo à instituição. Assim, de acordo com o levantamento realizado pelo Tribunal de Contas da União (TCU) sobre a Gestão dos Riscos nas Instituições da Administração Pública Federal, a presente IFES na qual o PROGERIS foi aplicado alcançou o menor índice de Fragilidade de Controles na Gestão de Riscos e Controles Internos $(0,34)$, sendo este o melhor índice dentre as 105 de instituições de ensino participantes do levantamento (TCU, 2018).

Essa avaliação é feita a partir de um questionário aplicado aos dirigentes e profissionais de Gestão de Riscos e Auditoria das instituições. O índice avalia se as instituições estão gerenciando seus riscos, quais são as ferramentas e controles estabelecidos e qual o nível de fragilidade dos mesmos frente as ameaças enfrentadas na realização de suas atribuições e missão institucional. Diante dessa análise, o TCU objetiva diagnosticar as fragilidades e fortalecer a GR dessas organizações.

Contudo, observa-se que o framework proposto também pode ser aplicado em outros contextos, públicos e privados, por se tratar de uma metodologia simples e pouco burocrática. Pontua-se, ainda, que a implantação da GR em um único setor com o uso do PROGERIS leva, em média, 4 meses.

\section{CONSIDERAÇÕES FINAIS}

O presente trabalho objetivou apresentar a aplicação de uma proposta de framework que foi desenvolvida à luz dos principais modelos de Gestão de Riscos, para o processo de gerenciamento de riscos de uma IFES. As percepções dos colabores dos setores da IFES que 
utilizaram o PROGERIS, bem como dados desse gerenciamento foram tratados e apresentados, respaldando os benefícios que o modelo proporciona no contexto onde ele é aplicado.

Pontua-se que pela sua simplicidade, etapas bem definidas e sustentadas pela literatura e prática, o PROGERIS pode ser adaptado e implementado em outros contextos de organizações públicas e privadas, facilitando todo o desenvolvimento e gerenciamento dos riscos corporativos.

Limitações da presente pesquisa foram identificadas quanto à grande quantidade de processos existente na IFES e baixa quantidade de facilitadores para conduzir as oficinas, fazendo com que somente os processos primários dos setores fossem contemplados com a GR. Nesse sentido, os riscos existentes em processos de apoio e os riscos gerenciais ainda não foram monitorados e controlados. Além disso, apesar das capacitações ofertadas aos atores dos processos, houve dificuldades por parte deles na compreensão da classificação e atribuição de valores para probabilidade e impacto do evento de risco. Por fim, notou-se morosidade na execução dos planos de ação pós-oficinas, retardando assim o início da gestão de riscos residuais.

Contudo, proposições de trabalhos futuros devem levar em consideração: estudo sobre o monitoramento e controle dos riscos; um estudo aprofundado sobre a integração que o presente framework de Gestão de Riscos e Gestão de Projetos; e pesquisas sobre a dinâmica do engajamento das equipes com a utilização no modelo.

\section{REFERÊNCIAS}

ABPMP - Association Of Business Process Management Professionals International. Guia para gerenciamento de processos de negócios - Corpo Comum de Conhecimentos (BPM CBOK®). [S.1.]: [s.n.], v. 3.0, 2013.

BOLZAN, C. I. M. Excelência em gestão universitária: um estudo de caso em uma instituição federal de ensino superior. Dissertação (Mestrado em Engenharia da Produção) - Curso de Pós-Graduação em Engenharia de Produção, UFSM. Santa Maria. 2006.

CAREGNATO, Rita Catalina Aquino; MUTTI, Regina. Pesquisa qualitativa: análise de discurso versus análise de conteúdo. Texto Contexto Enfermagem, Florianópolis, v. 4, n. 15, p.679-684, 2006.

COSO - Committee Of Sponsoring Organizations of the Treadway Commission. enterprise risk management - Integrated Framework. [s.1.], 2007.

GIBBS, Graham. Análise de dados qualitativos. Porto Alegre: Artmed, 2009. 198 p. 
GUN, Woo Choi; VIKRAM, Dhar; LUKE, Frey; HECTOR, Herrada; KENNY, Wunder, Janet Zaeiter; JAMES, Swanke. assessing the value and challenge of erm implementation. Insights, [s.1.], p.1-8, set. 2013.

HAMILTON, D. F. et al. Assessing treatment outcomes using a single question. The Bone \& Joint Journal, [s.1.], v. 96-, n. 5, p.622-628, maio 2014. British Editorial Society of Bone \& Joint Surgery. http://dx.doi.org/10.1302/0301-620x.96b5.32434.

ISO - International Standard. Iso 31000: Risk management — Principles and guidelines, [s.1.], 2009.

KAPLAN, Robert S.; MIKES, Anette. Managing risks: a new framework. harvard business review, [s.1.], p.1-20, jun. 2012.

KERSTIN, Dornberger; SIMONE, Oberlehner; NICOLE, Zadrazil; LEHNER, Othmar M. Challenges in implementing enterprise risk management. Acrn Journal Of Finance And Risk Perspectives, [s.1.], v. 3, n. 3, p.1-14, nov. 2014.

LIMA, O. F. de; LEITE, J. P. Aplicabilidade dos conceitos da produção enxuta (lean production) no processo de licitação pública. In: Encontro Nacional de Engenharia da Produção, 36, Anais do Encontro Nacional de Engenharia de Produção ENEGEP e ICIEOM (p. 1 - 14). Belo Horizonte: Abepro, 2011.

IRM - Institute of Risk Management. A risk practitioners guide to ISO 31000. N: IRM, 2018.

HILLSON, David. Developing effective risk responses. In: Proceedings Of The 30th Annual Project Management Institute 1999 Seminars \& Symposium Philadelphia, 30., 1999, Pennsylvania. Proceedings... Pennsylvania: Toolbar, 1999.

LIKER, Jeffrey K. O modelo toyota de produção: 14 princípios de gestão do maior fabricante do mundo. Porto Alegre: Bookman, 2005.

Ministério Planejamento Ministério da Fiscalização, Transparência e Controle. Instrução Normativa ${ }^{\circ}$ 01, de 2016. Controles Internos, Gestão de Riscos e Governança no Âmbito do Poder Executivo Federal, 2016.

MIRANDA, Rodrigo Fontenelle. Implementando a gestão de riscos no setor público. Belo Horizonte: Fórum, 2017.

MORAIS, Macelly Oliveira; PINTO, Antonio Carlos Figueiredo; KLOTZLE, Marcelo Cabus. Scenario analysis in the BNDES experience: integrating operational risk management with the measurement of capital. Revista Contabilidade \& Finanças, [s.1.], v. 29, n. 77, p.283-296, maio 2018. FapUNIFESP (SciELO). http://dx.doi.org/10.1590/1808-057x201804730.

PAPADAKI, M. et al. Essential factors that increase the effectiveness of project/programme risk management. Procedia - Social And Behavioral Sciences, [s.1.], v. 119, p.921-930, mar. 2014. Elsevier BV. http://dx.doi.org/10.1016/j.sbspro.2014.03.103.

PENHA, José Carlos; PARISI, Cláudio. Um caminho para integrar a gestão de riscos à controladoria. In: Congresso Internacional de Custos, 9., 2005, Florianópolis. Anais... . Florianópolis: Congresso Internacional de Custos, 2005. p. 2 - 16.

POWER, Michael. The risk management of everything. The Journal Of Risk Finance, [s.l.], v. 5, n. 3, p.58-65, mar. 2004. Emerald. http://dx.doi.org/10.1108/eb023001. 
PRIOTEASA, Adina-liliana; CIOCOIU, Carmen Nadia. Challenges in implementing risk management: a review of the literature. In: International Management Conference, 11., 2017, Bucharest. Proceedings... Romania: International Management Conference, 2017. p. 972 - 980.

RAZ, T; MICHAEL, e. Use and benefits of tools for project risk management. International Journal of Project Management, [s.1.], p.9-17, abr. 2001.

RICHARDSON, R. J. (2008) Pesquisa social: métodos e técnicas. $3^{\text {a }}$ ed. São Paulo: Atlas

ROBERTS, Alexander; WALLACE, William; MCCLURE, Neil. Strategic risk management. [s.1.]: Edinburgh, 2012.

ROSTAMI, Ali. Tools and Techniques in Risk Identification: A research within smes in the uk construction industry. Universal Journal Of Management, [s.1.], v. 4, n. 4, p.203-210, abr. 2016. Horizon Research Publishing Co., Ltd.. http://dx.doi.org/10.13189/ujm.2016.040406.

SARKA, Debasis; DUTTA, Goutam. A framework of project risk management for the underground corridor construction of metro rail. Indian Institute Of Management Ahmedabad, p.1-28, fev. 2011.

SANTOS, José Glauber Cavalcante dos; COELHO, Antonio Carlos. Value-relevance of disclosure: risk factors and risk management in Brazilian firms. Revista Contabilidade \& Finanças, [s.1.], v. 29, n. 78, p.390-404, 26 jul. 2018. Fap UNIFESP (SciELO). http://dx.doi.org/10.1590/1808-057x201806150.

SAMPIERI, R. H., Collado, C. F. \& Lucio, M. D. P. B. Metodologia de pesquisa. $5^{\text {a }}$ ed. Porto Alegre: Penso, 2013.

SILVA, Bruno José Pereira. Proposta de modelo de gestão de riscos para uma ifes visando a realização de auditoria baseada em riscos. 2015. 188 f. Dissertação (Mestrado) - Curso de Pós Graduação em Processos Institucionais da Ufrn, Universidade Federal do Rio Grande do Norte, Natal/rn, 2015.

Tribunal de Contas da União. auditoria exposição da administração pública federal a fraude e corrupção. Brasil, 2018.

TSERNG, H. Ping et al. A study of ontology-based risk management framework of construction projects through project life cycle. Automation In Construction, [s.1.], v. 18, n. 7, p.994-1008, nov. 2009. Elsevier BV. http://dx.doi.org/10.1016/j.autcon.2009.05.005.

TUMMALA, Rao; SCHOENHERR, Tobias. Assessing and managing risks using the Supply Chain Risk Management Process (SCRMP). Supply Chain Management: An International Journal, [s.l.], v. 16, n. 6, p.474-483, 27 set. 2011. Emerald. http://dx.doi.org/10.1108/13598541111171165.

UFRN - Universidade Federal do Rio Grande do Norte. Resolução no 076/2017-consad.

WEINSTEIN, Genna; MCWHA, Jena Prideaux. Implementing enterprise risk management. Washington: Education Advisory Board, 2008.

YIN, Robert K. Estudo de caso: planejamento e métodos. Porto Alegre: Bookman, 2005. 